\title{
MISE-EN-ABYME DER REZEPTION. ZU DEN FRÜHKRITIKEN DES CHASARISCHEN WÖRTERBUCHS
} Institute for Literature and Art, Belgrade

\section{AUF DEUTSCH}

\begin{abstract}
Mit implizitem Blick auf rezeptionstheoretische Fragen befasst sich die Arbeit mit der zeitlich frühesten Aufnahme des Chasarischen Wörterbuchs von Milorad Pavić im deutschsprachigen Raum. Untersucht werden die Erwähnungen des Romans und seines Autors in deutschsprachigen Printmedien vor der Veröffentlichung der deutschen Romanübersetzung, mit Ausblick auf die spätere Entwicklung der Rezeption innerhalb der Zeitungskritik. Die detaillierte Nachzeichnung der Inhalte dieser Frühphase soll zeigen, in welchem Maße die darin geäußerten Urteile über den Roman sowie das präsentierte schriftstellerische und persönliche Profil des Autors die gesamte nachfolgende Aufnahme des Chasarischen Wörterbuchs in der deutschsprachigen Tageskritik vorwegnehmen. Die Arbeit schließt mit einem Fragenkomplex über den Aussagewert dieser Schlussfolgerungen in Hinblick auf allgemeine Mechanismen literarischer Rezeption bzw. auf mögliche rezeptionsästhetische Verallgemeinerungen.
\end{abstract}

Schlüsselwörter: Milorad Pavić, Das Chasarische Wörterbuch, deutschsprachiger Raum, Mechanismen literarischer Rezeption, Rezeptionsgeschichte, Rezeptionsästhetik

Als Autor/Autorin mehrerer publizierter Arbeiten zum Thema der deutschsprachigen Rezeption von Milorad Pavić sieht man sich beim Gedanken an einen weiteren Beitrag zur Problematik mit der Berechtigungsfrage konfrontiert. Das Gefühl, alle oder nahezu alle relevanten Aspekte der Thematik bereits hinlänglich beleuchtet und öffentlich präsentiert zu haben, lässt die Unverzichtbarkeit einer weiteren Unternehmung in dieselbe Richtung bedenklich erscheinen. Die Frage nach der Begründung des eigenen Schreibens, die auch generell zum reflexiven Basisinventar des sogenannten wissenschaftlichen Arbeitens gehören sollte, stellt sich hier noch dringender.

Ausschlaggebend für die Entscheidung für einen weiteren Text zur selben Problematik war in diesem Fall der Eindruck, dass die hier vorgenommene Analyse der frühen Rezeption von Milorad Pavić und dem Chasarischen Wörterbuch in deutschsprachigen Medien über ihren unmittelbaren Gegenstand hinaus auch Allgemeineres über die Mechanismen literarischer Rezeption verraten könnte.

Die Veröffentlichung des Chasarischen Wörterbuchs auf Deutsch im Jahr 1988 verhalf Milorad Pavić über Nacht zu außerordentlichem Erfolg 
im deutschsprachigen Raum und verschaffte seinem Roman den Status eines Kultbuchs. Doch bevor im Februar 1988 die Hauptphase der tageskritischen Rezeption einsetzt, gestützt auf eine überwältigende Anzahl fast ausnahmslos affirmativer Rezensionen, finden sich - bereits ab 1985 - in deutschsprachigen Medien Meldungen zum Chasarischen Wörterbuch und seinem Autor. Blickt man zurück auf diese frühen Berichte, allesamt vor der Publikation des Romans auf Deutsch erschienen, so sticht darin ein Umstand heraus, der rezeptiongeschichtlich, möglicherweise auch rezeptionstheoretisch, mehr als belangvoll erscheint. Die frühesten Erwähnungen des Chasarischen Wörterbuchs und seines Autors in der deutschsprachigen Presse und im Werbematerial von Pavićs Hanser Verlag nehmen bereits alle relevanten Themenschwerpunkte vorweg, die in der darauffolgenden tageskritischen Rezeption des Romans und seines Autors (im Zusammenhang mit diesem Roman) ständig wiederkehren. Schon vor dem Erscheinen des Chasarischen Wörterbuchs auf Deutsch waren demnach in den Erstmeldungen zum Schriftsteller und seinem Werk fast alle Weichen für den späteren Rezeptionsverlauf gestellt. In diesem Sinne kann das Verhältnis zwischen Früh- und Hauptphase der Rezeption des Chasarischen Wörterbuchs möglicherweise als Fallbeispiel gelten, das zu allgemeineren Aussagen über rezeptionsgeschichtliche Prozesse und rezeptionsästhetische Phänomene führen könnte.

Die Entscheidung zur Veröffentlichung dieses Textes verweist darauf, dass seine Autorin nach anfänglichen Zweifeln die Gewissensfrage in Bezug auf die Legitimation des eigenen Schreibens zu ihrem Gunsten entschieden hat. Ob sie das darin implizierte Versprechen eingelöst hat, möge der Leser selbst entscheiden.

\section{Die Frühphase der Rezeption - Presseerwähnungen des Chasarischen Wörterbuchs vor 1988}

In eingeweihten Kreisen des deutschsprachigen Raums eilt der Ruhm des Chasarischen Wörterbuchs dem Werk voraus. Bereits im September 1985, etwa zweieinhalb Jahre vor Erscheinen der deutschen Übersetzung, findet sich ein Hinweis zum Roman in einer der auflagenstärksten deutschen Tageszeitungen, der Frankfurter Allgemeinen Zeitung. Der Kontext, in dem das Chasarische Wörterbuch apostrophiert wird, steht dabei auf verblüffende Weise im Einklang mit der Welt des „Lexikonromans“. Der ausführliche Artikel von Viktor Maier, „Der heilige und der historische Method“' verfasst anlässlich des Todesjahres des heiligen Method, behandelt ein Thema, das erstaunlich an den Duktus des Chasarischen Wörterbuchs erinnert: die in neueren Beiträgen zum Werk von Kyrill (bei Meier: "Cyrill“) und Method latent vorhandene Kontroverse, ob die Mission der Brüder „,in erster Linie unter religiösen oder aber unter kulturgeschichtlichen Aspekten zu betrachten sei“ (Maier 1985). 
Meier veranschaulicht damit die anhaltende Brisanz eines Themas, das auch elf Jahrhunderte nach den betreffenden historischen Begebenheiten die Gemüter erhitzt und spaltet. Als eines der religiösen und kulturellen Zentralereignisse des europäischen Mittelalters ist die Missionierung der slawischen Völker gleichzeitig ein Beispiel für die engste Verflechtung von religiösem und kulturellem Engagement mit politischer Agitation und politisch-institutionellem Machtkampf. Meiers Hauptaugenmerk gilt der christlichen Missionierung der Slawen, mit welcher Kyrill und Method als angesehene byzantinische Gelehrte von Kaiser Michael III beuftragt wurden. Ihre Sendung trug ganz offenbar neben religiösem auch Kulturcharakter, denn die Festigung der christlichen Glaubenslehre entwickelte sich innerhalb einer weitaus breiteren kulturellen Aktivität. Sie schloß u. a. Übersetzungen liturgischer Texte und der Bibel aus dem Griechischen ins Altkirchenslawische wie auch die Entwicklung der Glagoliza als der neuen, dem Slawischen gemäßen, Schrift ein. Auf diese Weise leisteten die Brüder aus Saloniki einen enormen Beitrag zur Integration vieler slawischer Völker in den byzantinischen Kulturraum. Doch Aspirationen auf die Eingliederung der Slawen in den eigenen Herrschafts- und Einflussbereich kamen auch von anderer Seite, so zum Beispiel von den „fränkischen Glaubensboten“ (Maier 1985) im Falle der slawischen Völker des Großmährischen Reiches. Die Rivalitäten ergaben sich also zu einem Großteil auch aus der formal noch nicht durchgeführten, aber tatsächlich existierenden Spaltung des Christentums in eine Ost- und Westkirche ${ }^{1}$. Meier führt deutlich vor Augen, wie die Slawen-Mission von Kyrill und Method in den politischen Kampf zwischen Byzanz und den Franken bzw. Ost- und Westchristentum um staats- und kirchenpolitische Einflusssphären eingebettet war. Der missionarische Eifer beider Seiten war darauf ausgerichtet, den identitätsmäßig noch formbaren slawischen Raum und dessen Völker für sich zu gewinnen.

Hochaktuell und konfliktträchtig erscheint der Gegenstand, Meier zufolge, weil Spuren der jahrhundertealten Spannungen noch im Jahre 1985 vernehmbar sind:

Die Stadt Saloniki [...] sieht die Mission als ihren und des ganzen Hellenismus Beitrag zur Erleuchtung der slawischen Barbarenwelt; zumindest möchte sie der Ostkirche dabei den gebührenden Rang zuweisen. In katholischen Kreisen des Westens betonte man mit einem gewissen Hang zur Ausschließlichkeit, Cyrill und Method hätten sich nur als Glaubensboten betrachtet, und seien vom Gefühl einer „einheitlichen, wahrhaft katholischen Kirche“ durchdrun-

1 Vgl. dazu folgende Erläuterung Maiers: „Cyrill und Method, beide hochgebildet und diplomatisch geschult, zeigten mit ihrer ganzen Handlungsweise, daß sie sich bewußt waren, in einem außerordentlich heiklen Szenarium zu agieren. Zwar war damals die Kirchenspaltung offiziell noch nicht vollzogen, aber tatsächlich bestand sie. Die Kirchenfrage wiederum war eingebettet in ein politisches Spannungsfeld mit zwei und oftmals sogar mehreren Polen. Die Brüder konnten hier nicht einmal verbindend wirken, sondern höchstens lavierend, und oft mußten sie sich in direkten, harten Auseinandersetzungen behaupten“ (Maier 1985). 
gen gewesen. Papst Johannes Paul II, slawischer Abstammung, brachte zwar in seine Enzyklika "Slavorum apostoli“ auch kulturgeschichtliche Momente ein, wurde deswegen aber kritisiert (Maier 1985).

Als Fazit der Untersuchung könnte man Maiers Bemerkung auffassen, der historische und der heilige Method würden im Jubiläumsjahr oft „als zwei verschiedene Persönlichkeiten“ (Maier 1985) erscheinen. Das Chasarische Wörterbuch wird als belletristischer Beitrag zur Debatte und als literarisches Zeugnis für das religiöse Profil von Kyrill und Method herangezogen:

An der glaubensbewegten Frömmigkeit der Brüder aus Saloniki ist nicht zu zweifeln; diese hatte sich schon bei der vorangegangenen Mission zu den Chasaren manifestiert. Der serbische Schriftsteller Milorad Pavić hat sich davon anregen lassen, unter dem Titel „Chasarisches Wörterbuch" einen in Wörterbuchform verfaßten Roman zu schreiben, der in Jugoslawien als „Roman des Jahres" ausgezeichnet wurde. Er schildert, unter Anspielung auf das heutige Jugoslawien, ein Land, in dem drei Religionen um den Sieg ringen (Maier 1985).

Maier markiert in diesem knappen Vermerk die religiöse Thematik, die auf konfessionelle Gegensätze Jugoslawiens Bezug nimmt, als zentral im Chasarischen Wörterbuch. Die etwas apodiktische Reduzierung des Romans auf die Glaubensproblematik ist hier natürlich kontextbezogen, da sich der Artikel mit der religiösen und/oder kulturgeschichtlichen Identität von Method (und Kyrill) befasst. Trotzdem könnte man gerade diese Verkürzung der Perspektive bedauern, finden sich doch im Chasarischen Wörterbuch Entsprechungen zum im Artikel angesprochenen Hauptdilemma. So reicht die Darstellung der Slawenapostel im Roman weit über deren religionsgeschichtliche Bedeutung für die slawischen Völker hinaus und bezieht auch ihre kulturgeschichtliche Rolle mit ein. Als Illustration sei hier nur auf die poetischen Ausführungen zur Entstehung der slawischen Schrift aus der Hand des heiligen Kyrill verwiesen. ${ }^{2}$ Auch sonst bezieht sich Pavić im Chasarischen Wörterbuch sehr gerne auf die latente Vernetzung von religiösen, kulturellen und ideologischen Diskursen und Akten. Sprache, Schrift, Glaube, Ideologie und Politik sind darin, ähnlich wie in der von Meier behandelten Thematik, unentwirrbar miteinander verflochten.

2 Beim Stichwort „Kyrillos“ im Roten Buch heißt es zur Entwicklung der slawischen Schrift: „Folgende Erzählung über die Entstehung des slawischen Alphabets überliefert Daubmannus. Die Sprache der Barbaren wollte sich in keiner Weise zähmen lassen. In einem schnellen, drei Wochen langen Herbst saßen die Brüder in der Zelle und versuchten vergeblich, die Buchstaben zu formen, die man später Kyrilliza nannte. Die Arbeit ging ihnen nicht von der Hand. [...] Da lenkte Methodios die Aufmerksamkeit seines Bruders auf vier Tonkrüge, die im Fenster ihrer Zelle standen, doch draußen, jenseits des Gitters.

,Wie würdest du an einen dieser Krüge kommen, wenn deine Tür verriegelt wäre?' fragte er. Konstantin zerschlug einen der Krüge, holte die Scherben Stück für Stück durch das Gitter in die Zelle und setzte dort den Krug wieder zu einem Ganzen zusammen, während er ihn mit seinem Speichel und dem Lehm des Fußbodens unter seinen Füßen leimte.

So hielten sie es auch mit der slawischen Sprache, sie zerschlugen sie in Stücke, holten sie durch das Gitter der kyrilischen Buchstaben in ihre Münder und leimten die Scherben mit ihrem Speichel und dem griechischen Lehm unter ihren Fußsohlen..." (Pavić 1991, 92). 
Vor der Veröffentlichung der deutschen Übersetzung wird das Chasarische Wörterbuch noch einmal, und zwar ganz unmittelbar, in einen wissenschaftlichen Kontext einbezogen. Es ist dieses Mal Pavić selbst, der einen direkten Konnex zwischen seinem Roman und der Literaturwissenschaft anschaulich macht. Der Anlaß ist ein internationales Symposium zur slawischen Barockliteratur an der Universität Mannheim, an dem auch Pavić teilnimmt. Darauf anschließend präsentiert Walter Kroll im Uni-Report der Mannheimer Universität eine Zusammenfassung der Tagungsergebnisse (Vgl. Kroll 1986: 7). Von den zahlreichen Vorträgen renommierter Barockexperten markiert Kroll einzig Pavićs Diskussionsbeitrag als „unorthodox“ (Vgl. Kroll 1986: 7). Die Qualifikation bezieht sich jedoch nicht auf die inhaltliche Seite des Gegenstands „serbische Barockliteratur“ in Pavićs Aufsatz, sondern auf das unkonventionelle Verfahren von dessen Illustration. Pavić verschränkt nämlich auf originelle Art und Weise beide Seiten seiner beruflichen Orientierung, indem er die Barockschicht des Chasarischen Wörterbuchs im Kontext der serbischen Barocktradition situiert und darauf aufbauend die Genese der serbischen Barockliteratur erläutert.

Die letzte unter den frühesten Erwähnungen des Romans erfolgt Ende 1986 in der Mittelbayerischen Zeitung. In einem Kurzbericht informiert darin Vladimir Ulrich über Pavićs Lesung aus dem Chasarischen Wörterbuch in der Regensburger Stadtbücherei, veranstaltet anlässlich der 6. Internationalen Regensburger Literaturtage (Vgl. Ulrich 1986). Ulrich verweist zu Beginn auf die zweifache Beziehung des Autors zu Regensburg - auf seine Gastprofessur an der dortigen Universität im Wintersemester 1986/87 (die Lesung fällt also in diese Periode hinein) sowie auf den Umstand, dass das Chasarische Wörterbuch zwischen 1978 und 1983 in Belgrad und Regensburg geschrieben wurde. Darüber hinaus wird die steil ansteigende Erfolgskurve des Romans im In- und Ausland mit 50000 verkauften Exemplaren in Jugoslawien und laufenden Übersetzungen in zwölf Fremdspachen erwähnt. Der Kritiker beurteilt den Roman als ,interessantes formales Experiment“ und kündigt dessen deutsche Übersetzung an, die „vermutlich im nächsten Jahr bei Piper“ (Ulrich 1986) erscheinen solle. Dass Ulrich zu jenem Zeitpunkt sowohl mit dem Erscheinungsjahr als auch mit dem Verlag falsch liegt, ist, wie man aus einer späteren Rezension desselben Kritikers erfährt, auf den Umstand zurückzuführen, dass der Roman tätsächlich zunächst dem Piper Verlag zur Publikation angeboten worden war. Dieser habe es jedoch fälschlicherweise anstatt als belletristische Prosa als stark korrekturbedürftige mystische Literatur eingeschätzt, woran letztlich wohl die Verhandlungen zwischen Verlag und Autor gescheitert sein müssen (Vgl. Ulrich 1989).

Wichtig erscheint in diesem Kontext vor allem Ulrichs Hinweis auf die gattungsmäßige Heterogenität und Unbestimmbarkeit des Werks, das der Kritiker als „Abenteuerroman, eine Sammlumg von Erzählungen, Märchen und Legenden, ein Gedichtband, eine historische Studie, ein 
kabbalistisches Handbuch - und vieles mehr" (Ulrich 1986) bezeichnet. Diese recht frei formulierte Genrequalifikation wird in der späteren Zeitungskritik auffallend häufig - teilweise unter wörtlichen Anleihen bei Ulrich - zur Charakterisierung des Romans herangezogen.

\section{Ankündigungen im Vorfeld der Romanerscheinung}

Bärbel Schulte übersetzt das Chasarische Wörterbuch auf Eigeninitiative und bietet das Manuskript zunächst ohne Erfolg verschiedenen Verlagen zur Publikation an. Schließlich findet der Münchner Carl Hanser Verlag Interesse am Roman und bringt ihn im Februar 1988 heraus. Pavićs deutscher Verlag startet unmittelbar danach eine Marketingkampagne für das Werk und organisiert Buchpräsentationen und Lesungen in mehreren deutschen, österreichischen und Schweizer Städten: Wien, Zürich, Berlin, Regensburg, München, Passau und Köln (Vgl. Цидилко 2006: 611).

Dank den besprochenen frühen Presseerwähnungen sind Pavić und sein Roman zu jenem Zeitpunkt keine gänzlichen Unbekannten im deutschsprachigen Kulturkreis. Doch die Kenntnis über den Autor und sein Werk beschränkt sich zweifellos auf das akademisch-universitäre Umfeld (und darin namentlich auf die deutschsprachige Slawistik), in dem Pavić vorrangig als angesehener Literaturwissenschaftler wahrgenommen wird. Gerade das hohe Renommee des Autors in der wissenschaftlichen Forschung mag in Teilen der Fachwelt sogar Verwunderung über Pavićs „literarische Wende“ ausgelöst haben. Das scheint zumindest die folgende Bemerkung eines anonymen Kritikers der Mittelbayerischen Zeitung zu signalisieren: „Wer in Deutschland bis zu diesem Zeitpunkt [bis zur Publikation des Romans auf Deutsch, M. M.] bereits etwas von Milorad Pavić gehört hatte, der zuckte endgültig zurück, wenn er vernahm, dieser slawische Tausendassa habe nun ein Wörterbuch ,gebaut'“ (Anonym 1 1988). Einen ähnlichen Überraschungseffekt löst übrigens die vom raschen Publikumserfolg begleitete Romanerscheinung auch in Jugoslawien aus. Dort gilt Pavić als anerkannte Autorität auf dem literaturwissenschaftlichen Feld, während seine literarische Produktion bis zur Veröffentlichung des Chasarischen Wörterbuchs von der Leserschaft weitgehend unbeachtet bleibt. Wie er selbst gerne hervorzuheben pflegte, war er bis zum Jahr 1984 der am wenigsten gelesene Autor seines Landes und nach 1984 der meistgelesene (Vgl. Павић 2005: 7).

Pavićs nahezu völlige Anonymität in breiteren Kreisen der deutschsprachigen Leserschaft mag mit ein Grund dafür gewesen sein, dass im unmittelbaren Vorfeld der deutschen Romanveröffentlichung zwei Publikationen mit Vorstellungs- und Werbecharakter die Aufmerksamkeit auf das Chasairsche Wörterbuch lenken.

Am 30. Jänner 1988 erscheint in der österreichischen Presse der Artikel „Ökumenischer Traum“ von Zdzişlaw P. Gwozdz, hervorgegangen 
aus einem in Belgrad geführten Gespräch mit dem serbischen Schriftsteller. Der Journalist bietet einen ersten Einblick in die Welt des, wie es heißt, „geheimnisvolle[n]“ (Gwozdz 1988) „Lexikonromans“, möchte aber darüber hinaus auch ein allgemeineres Bild von Pavić als Autorfigur und Privatperson vermitteln, indem er vor allem einige Dominanten seiner Weltanschauung identifiziert.

Gwozdz setzt beim sich abzeichnenden Kultstatus des Buchs an und parallelisiert es mit Umberto Ecos damals schon weltberühmtem Debütroman Der Name der Rose. Wie auch im späteren Rezeptionsverlauf sehr häufig der Fall, sichtet der Journalist in den beiden Werken thematische und formale Berührungspunkte und präsentiert sie deshalb als fiktive Konkurrenten um den größeren literarischen Erfolg: „Wird das ,Chasarische Wörterbuch', ein ,Lexikonroman' (Untertitel), ein Welterfolg wie Umberto Ecos ,Name der Rose'? Dafür spräche ein neuerwachtes Interesse am Mittelalter und an enträtselbaren Montagen literarischer Altertümer“ (Gwozdz 1988).

Wie im Untertitel angekündigt, kommt der Rezensent zunächst auf das Magisch-Rätselhafte im Chasarischen Wörterbuch zu sprechen, das sich gleichermaßen in der Wirkungskraft der lexikalischen Form wie in thematischen und inhaltlichen Grundzügen des Werks manifestiere. Gwozdz attestiert dem Roman „eine gewisse Universalität“ aufgrund der magisch-verschwörerischen Verbundenheit, die sich als Folge der Lektüre zwischen den Lesern einstelle: „Jene, die die Lektüre des ,Wörterbuchs hinter sich haben, können miteinander ,kommunizieren', einander etwas ,mitteilen“". Leider ohne erhellende Worte über die Eigenart dieser angeblich spezifischen Kommunikationsebene geht der Kritiker zum Lob der ästhetischen Wirkung des Romans über. Dieser vermittle durch die Fragmentarisierung der Form und die Beliebigkeit der Lektüreabfolge, „ein neues ,Lesegefühl", dem kaum jemand widerstehen kann" und befriedige dadurch den „Spieltrieb“ (Gwozdz 1988) des Lesers.

Etwas weiter im Text bringt Gwozdz das Gespräch auf die thematische Seite des Mysteriösen in seiner jenseitig-diabolischen Spielart: „Doch das Echo auf das ,Wörterbuch“ entspricht doch irgendeiner Sehnsucht nach dem Dunklen, Verborgenen, vielleicht Okkulten, ist das Buch doch voller Dämonen, Geister, Teufel und Vampire?" (Gwozdz 1988). Die Frage lässt der Journalist (bzw. Pavić, an den sie gerichtet war) jedoch unbeantwortet im Raum stehen - leider, könnte man meinen, denn gerade die esoterisch-metaphysischen Bezüge des Romans bleiben auch später weitgehend außerhalb des Blickfelds der Zeitungskritik.

Eingehender widmet sich der Kritiker der Frage nach dem Symbolgehalt bzw. der konkreteren Referenz des Chasaren-Themas, womit er einen in der späteren Pavić-Rezeption gängigen und gewichtigen Gegenstand anschneidet. Es geht um die Frage, inwieweit Pavić im Identitätsverlust der Chasaren ganz bestimmte Sinnbilder zu schaffen beabsichtigt hatte. 
Gwozdz lässt hierzu den Schriftsteller selbst ausführlich zu Wort kommen. Wie Pavić im Klartext erläutert, wollte er mit der chasarischen Metapher ${ }^{3}$ zunächst einen konkreten Beitrag zur serbischen Erinnerungskultur leisten: eine Rückbesinnung auf die Verankerung der serbischen literarischen Tradition im byzantinischen Kulturraum:

Ich wollte mit der Geschichte der Chasaren, einem Volk, über dessen Herkunft, Aufstieg und Niedergang die Historiker und Literaturwissenschaftler streiten, durchaus eine Parallele schaffen. Die serbischen Schriftsteller sind in ihrem Wesen noch immer byzantinisch, ich auch. Dieses Ambiente erlebe ich als mein eigenes, das „Wörterbuch“ ist eine Suche nach dem eigenen Selbstverständnis und der eigenen Herkunft (Gwozdz 1988).

An keiner anderen Stelle verweist Pavić bei der Eigeninterpretation des Romans so deutlich auf Byzanz als Erinnerungsort der serbischen Kultur und Literatur. Doch diese Rückbesinnung bezieht er fast im selben Atemzug in einen noch breiteren kulturgeschichtlichen und (gesellschafts)politischen Kontext ein:

Ein [...] Problem betrifft, ähnlich wie die Chasaren, meine eigene Nation. Ein kleines Volk zwischen großen Ideologien, Religionen und Mächten, das diese Ideologien und Religionen sowie die Macht nicht teilt. Die Chasaren, ein Volk und zugleich ein Modell, verschwanden von der Erde, doch sie haben sie reicher gemacht. Die Serben haben etwas mit den Deutschen gemeinsam - sie fühlen, daß Europa ihre Liebe nicht erwidert, daß sie nicht verstanden werden. Und die Serben haben doch eine fast tausendjährige Staatlichkeitstradition, eine fast 200 Jahre alte Erfahrung in Demokratie und Parlamentarismus, eine reiche Literatur und Kultur. Ich teile die Sorge um die nicht erwiderte Liebe kaum. Wir bieten der Welt neben Schach- und Tennisspielern von höchstem Rang auch Wissenschaftler, Maler, Architekten, Schriftsteller... Daß wir den heutigen Kriterien der Ökonomie und Politik nicht entsprechen? Auch wenn unser Blick ins 21. Jahrhundert reicht, dürfen wir nicht für eine Handvoll Mikrochips unsere Kultur abschütteln (Gwozdz 1988).

In gedrängter Form sind hier Pavićs Grundgedanken zum Wechselverhältnis zwischen Ideologie, Religion und kultureller Identität wiedergegeben, die in seinen späteren öffentlichen Stellungnahmen (teilweise mit deutlich größerem Enthusiasmus und verstärkter Rhetorik) ständig wiederkehren und auch in der Rezeption auf verschiedene Weise ihren Niederschlag finden. Vor allem wird die Analogie zwischen Chasaren und Serben später gerne als Interpretationsstütze herangezogen, um auf das Romanthema des drohenden kulturellen Indentitätsverlustes kleiner Völker (des serbischen, aber nicht nur des serbischen) im Machtkampf großer ideologischer und religiöser Systeme zu verweisen.

Hiermit unmittelbar verbunden ist Pavićs zweite Argumentationslinie. Ebenfalls durch eine Parallele zu den Chasaren ist es ihm ein An-

3 Das Syntagma "chasarische Metapher" steht in dieser Arbeit für das metaphorische bzw. symbolhafte Potenzial des im Roman thematisierten chasarischen Identitätsverlustes. 
liegen, die Bedeutung der Serben als kleinem Volk mit beachtenswerter politischer und kultureller Tradition hervorzukehren. Die Schlüsselstelle der zitierten Passage scheint dabei der Gedanke von der nicht erwiderten Liebe Europas zu Serben und Deutschen zu sein. Die Serben würden sich von Europa trotz ihrer relativen kulturellen Relevanz nicht im ausreichenden Maße anerkannt fühlen. Der Verweis auf die Deutschen könnte sich hingegen im Gesprächsjahr 1988 auf die Skepsis einiger europäischer Großmächte - Frankreich, Großbritannien - gegenüber deutschen Wiedervereinigungsbestrebungen beziehen. Dass es Pavić in der gesamten Argumentation vor allem um Erhaltung und Diversität kultureller Formen in Europa geht, lässt sich am Ende des Zitierten vernehmen, wo die Existenz zweier ungleicher politisch-ökonomischer Systeme des damaligen Europa (Kapitalismus vs. Sozialismus) nicht als Hindernis für kulturbewahrendes Vorgehen betrachtet wird.

Das Stichwort kulturelle Diversität leitet auch zum Themenschwerpunkt des Ökumenismus aus dem Artikeltitel über. Gwozdz erklärt zwar in der Einleitung „religiöse Toleranz“ mit Prägnanz zum „Hauptthema“ (Gwozdz 1988) des Romans, kommentiert diese aber im Artikel nicht weiter. Stattdessen macht er einen impliziten Brückenschlag zwischen weltanschaulichen Konzepten bzw. politischer Grundhaltung und literarischem Schaffen des Autors. Die Skizzierung des „ökumenischen Traums“ als Pavićs gesellschafts- und kulturpolitische Gesinnung überlässt Gwozdz wieder dem Autor persönlich:

Die Vielfalt ist unser Reichtum. In Griechenland und in Serbien ist die Tradition des Geistes auf Nationales gerichtet. Vielleicht deswegen, weil unsere Kirchen autokephal - national und unabhängig - waren und sind. Im Westen überwiegt der Internationalismus. Doch ich sehe die Chancen der Zukunft Europas weniger in den offiziellen Dialogversuchen der Staaten, Regierungen oder Kirchen. Ich sehe sie vielmehr in dem individuellen, inoffiziellen Austausch. Mein ,Chasarisches Wörterbuch' ist nicht zuletzt von einem weitgefaßten ökumenischen Traum getragen, dem Traum, daß wir unsere Unterschiede überbrücken und uns von allem befreien, was uns teilt, ärmer macht und voneinander verschließt" (Gwozdz 1988).

Pavić engagiert sich hier demnach für eine Ökumene, die nicht nur Religionsvielfalt und Vermittlung zwischen konfessionellen Gruppen voraussetzt, sondern im übertragenen Sinne auf transnationale Verbindungen und Verflechtungen auf kulturellem Terrain abzielt. Zieht man also die erweiterte Semantik von Ökumene bei Pavić in Betracht, könnte man statt von „religiöser“ eher von „Kulturtoleranz" sprechen. Damit schließt sich der argumentative Kreis von existentieller Bedrohung kleiner Kulturen, erhoffter kultureller Diversität Europas und religiöser Toleranz. Pavić sieht diese Phänomene in unmittelbarem Zusammenhang und formuliert darauf aufbauend zwei eng zusammenhängende kulturideologische Grundgedanken. Sein eindringliches Plädoyer für religiöse Toleranz und kulturelle Diversität ist im Allgemeinen auf die Überwindung 
des Ost-West-Gegensatzes gerichtet, im Konkreten aber auf die (Wieder) Verankerung des Ostens im westlichen Kulturbewusstsein. Daher auch die im Text - bei aller expliziten Verneinung des Autors - unüberhörbare Besorgnis, die serbische Kultur, und allgemeiner die osteuropäischen Kulturkreise, könnten nach dem Modell des chasarischen Identitätsverlustes auf lange Strecken aus dem westeuropäischen Kulturbewusstsein ausgeblendet bleiben.

Wichtig erscheint vor allem, dass Gwozdz dem Leser nahelegt, Pavićs ökumenische Gedanken schlagen sich als Religionstoleranz im Chasarischen Wörterbuch nieder, sogar als thematischer Kernpunkt des Romans. Mit anderen Worten, zwischen ideologischer Grundhaltung bzw. politischen Überlegungen des Schriftstellers und seiner Werkpoetik wird ein Gleichheitszeichen gesetzt.

Ein Großteil von Gwozdz' Artikel wird auch in die vom Pressedienst des Carl Hanser Verlags publizierte Informationsbroschüre zum Chasarischen Wörterbuch aufgenommen, die ebenfalls im Vorfeld der Veröffentlichung der deutschen Übersetzung (oder unmittelbar danach) erscheint (Vgl. Hanser Presse Dienst 1988) ${ }^{4}$. Das Heft macht auf acht Seiten Text mit den historischen Chasaren, mit Biographischem zu Pavić, mit Struktur und Poetik des „Lexikonromans“, sowie mit den Anfängen seiner Rezeption in Serbien bzw. Jugoslawien vertraut. Die Publikation des Pressedienstes ist als Erstorientierung für potenzielle Rezensenten gedacht, hat jedoch neben dem Auskunft- auch offenkundigen Werbecharakter.

Im dreiseitigen Hauptteil der Broschüre informiert Pavićs deutsche Übersetzerin Bärbel Schulte im Beitrag „Phantastische Reise in die Vergangenheit" (Hanser Presse Dienst 1988: 3) über den Autor und das Chasarische Wörterbuch. Wie schon im Titel signalisiert, legt Schulte den Akzent ganz auf Phantastik als Erkennungsmerkmal von Pavićs literarischem Schaffen. Sie stellt ihn zunächst als unkonventionellen Autor vor, der die Eigenschaft, gleichermaßen als Schriftsteller und Wissenschaftler betätigt zu sein, zwar mit renommierten zeitgenössischen Autoren teile (womit wieder die Anspielung auf Umberto Eco auf der Hand liegt), in seiner Heimat jedoch mit dieser "doppelte[n] Berufung" (Hanser Presse Dienst 1988: 3) eher einen Ausnahmefall darstelle. Dabei verdanke sich die Besonderheit des "Lexikonromans" gerade der Verbindung beider Professionen. Schulte betont vor allem, dass Pavić in beiden Bereichen seines doppelten Engagements aus dem Rahmen der zeitgenössichen literarischen bzw. literaturwissenschaftlichen Produktion fiel. So vermerkt sie seine trotz beachtlicher schriftstellerischer Tätigkeit anhaltende Au-

4 Das Erscheinungsdatum des Informationsheftes ist nicht bekannt. Es liegt auf jeden Fall nach dem 30. Jänner 1988, da die Broschüre den an diesem Tag veröffentlichten Presse-Artikel von Zdzişlaw P. Gwozdz in gekürzter Version übernimmt und auf die vollständige Fassung in der Presse verweist. Es ist auch anzunehmen, dass das Heft, seinem Informations- und Werbezweck entsprechend, vor oder kurz nach dem Erscheinen der deutschen Romanübersetzung publiziert wurde. 
Benseiterposition, die erst mit dem Erscheinen des Chasarischen Wörterbuchs in ihr Gegenteil umgekehrt wurde und erklärt diese ganz richtig durch poetologische und genremäßige Unangepasstheit des Autors an den vorherrschenden literarischen Mainstream Serbiens bzw. Jugoslawiens: „In der nichtmimetischer Literatur feindlich gesonnenen Atmosphäre der ersten Nachkriegszeit war sich Pavić [...] darüber im klaren, längere Zeit für die Schublade zu schreiben" (Hanser Presse Dienst 1988: 3). Gleiches gelte für Pavićs wissenschaftliche Laufbahn, wo er ebenfalls „den nichtbegangenen Weg" (Hanser Presse Dienst 1988: 3) gewählt habe. Hier bezieht sich Schulte auf bahnbrechende Studien zum serbischen literarischen Barock, Klassizismus und der Frühromantik, mit denen der Wissenschaftler Pavić die kontinuierliche Entwicklung der serbischen Literatur vom Mittelalter bis in die Gegenwart aufgezeigt habe.

Schultes Hauptaugenmerk gilt Pavićs phantastischem Diskurs, dank welchem der serbische Schriftsteller bereits seit der Veröffentlichung seiner Erzählungen „zum bedeutendsten Vertreter phantastischer Prosa in seinem Lande" avanciert sei. Es ist das Anliegen der Übersetzerin, Typ und Eigenart von Pavićs Phantastik in Abgrenzung zu anderen geläufigen Varianten des Genres näher zu bestimmen. Demnach schreibe Pavić "keine Grusel-Phantastik, keine wissenschaftliche Utopie“ und ebenso keine phantastische Prosa, die als „Fluchtweg aus der Welt des Realen“ (Hanser Presse Dienst 1988: 4) gedacht ist. Seine Spielart des Phantastischen sei auch nicht auf Negation, sondern auf das Transzendieren der sinnlich erfahrbaren Wirklichkeit gerichtet. Die nähere Veranschaulichung überlässt Schulte dem Autor selbst, den sie ausführlich zitiert:

Phantastik muß weder Eskapismus bedeuten, noch ein Verschließen der Augen vor der Realität. Sie nährt sich in gleichem Maße von der Lebenswahrheit wie von Tausenden kleiner Wahrheiten dieses Lebens; doch müssen diese Wahrheiten sehr überzeugend und absolut mimetisch ausgerichtet sein, damit man plötzlich den Teppich unter den Füßen wegzuziehen vermag, um all das in Frage zu stellen, was bis dahin so minutiös mit der Ambition aufgebaut wurde, die sinnliche Wirklichkeit zu beschwören. Wenn nichtmimetische Literatur die Flucht vor der augenscheinlichen Realität bedeutet, dann ist mimetische Literatur die Flucht vor allem anderen (Hanser Presse Dienst 1988: 4).

Die sinnlich erfahrbare Realität wird in Pavićs phantastischer Optik also nicht bestritten und aus dem literarischen Verfahren ausgeschlossen, sondern dient als jene notwendige ontologische Ebene, die es auf der Suche nach weniger sichtbaren und schwieriger zugänglichen Realitätsebenen zu transzendieren gilt. Charakteristisch ist hier Pavićs relativ dürftige und schematische Einteilung in "mimetische“ und "nichtmimetische“ Narration. In ihr hallt seine häufig vorgebrachte Kritik am Erbe der realistischen Prosa des 19. Jahrhunderts wider, deren Überwindung sich die zeitgenössische Literatur zur Hauptaufgabe machen solle und die er im eigenen Schaffensprozess anstrebe. Wie er in einem späteren Interview 
mit Mirjana Wittmann im Deutschen Allgemeinen Sonntagsblatt zu verstehen gibt, geht es ihm vor allem um die Umkehrung des festgefahrenen Primats in der Relation Literatur - Realität:

[...] in Jugoslawien wurde ich oft gefragt, wieviel darin [im Chasarischen Wörterbuch, M. M.] Geschichte und Wahrheit und wieviel Phantasie und Dichtung ist. Meine Antwort darauf lautet, daß es nicht darum geht, aus der Wahrheit Literatur zu machen, sondern aus der Literatur Wahrheit zu gewinnen. Das ist ein großer Unterschied im Verfahren. Der Roman des 19. Jahrhunderts, von dem die meisten heutigen Autoren zehren, befindet sich in einer großen Krise. Wir kommen nicht weiter, wenn wir fortfahren, dieses abgenutzte Muster weiter zu pflegen. Man muß mit dem Klischee des überlieferten realistischen Romans brechen (Wittmann 1988).

Das Gegensatzpaar „mimetisch - nichtmimetisch“ bezieht sich in Pavićs Ausführungen zumeist auf die Relation realistische - phantastische Gattungsformen. Nichtmimetische (hier phantastische) Literatur ist also dem Autor zufolge in genau jenem Maße „Flucht“ vor unmittelbarem Realitätsbezug, in dem realistische Diskurse „Flucht“ bzw. absichtliches Ignorieren tieferer und nicht unmittelbar zugänglicher Wirklichkeitsniveaus bedeuten. Mit der Wiedergabe eines weiteren autopoetischen Kommentars des Schriftstellers unterstreicht Bärbel Schulte die Eigenart von Pavićs Phantastik als Öffnung zu genau diesen tieferen Wirklichkeitsebenen, $\mathrm{zu}$ jenseits des sinnlich Erfahrbaren liegenden „Welten“:

Ich hatte das Gefühl, daß es zuweilen unerträglich langweilig ist auf der Welt, daß wir - fassen wir die Welt in gewohnter Weise buchstäblich auf - von ihr nicht übermäßig viel empfangen und daß wir uns in der Tat anstrengen sollten, ein wenig hinter die Dinge zu schauen, hinter die Erscheinungen, hinter die Membrane des Realen, wo vielleicht doch eine den Menschen unbekannte Welt existiert. Schließlich werden wir beständig damit konfrontiert, daß diese Welt Schritt für Schritt entdeckt wird. Es ging also um das Empfinden, auch die Literatur habe diesen allgemeinen Bemühungen etwas hinzuzufügen: eben damit befassen sich ja auch die Wissenschaftler, unaufhörlich zerstören sie die Membrane des Realen, nur, das ihnen das niemand nachträgt (Wittmann 1988).

Mit ironischem Seitenhieb auf die wissenschaftliche Forschung, erklärt Pavić auch die sinnlich erfahrbare Realität zur instabilen und variablen Kategorie - ein Beweggrund mehr für die Literatur, selbst „hinter die Erscheinungen“ zu blicken. Bei all dem bleiben seine Formulierungen jedoch kryptisch und allgemein genug, um eine exakte Wesensbestimmung jener „dem Menschen unbekannte[n] Welt“, die sich hinter der Membrane der empirischen Wirklichkeit offenbare, zu ermöglichen. Mit Blick auf das Chasarische Wörterbuch ließen sich hinter diesen Verweisen etliche Formen des Transzendierens sinnlicher Erfahrung ausmachen: Metaphysisches wie Okkultes, Unbewusstes, Onirisches, Irrationales u. a. Doch die relative Schattenhaftigkeit von Pavićs autoreferenziellen 
Kommentaren zum Chasarischen Wörterbuch und zum literarischen Schaffen als solchem mag mit ein Grund dafür gewesen sein, dass in der tageskritischen Diskussion einige der angedeuteten unbekannten Welten stärker aktzentuiert (so zum Beispiel Traumhaftes, Irrationales und Unbewusstes) und andere (Metaphysisches und Okkultes) weitgehend vernachlässigt wurden. Im Anschluß äußerst sich Schulte lobend über Pavićs Virtuosität „Wissenschaftliches' auf den Kopf zu stellen, spielerisch zu verwandeln oder zu untergraben" (Hanser Presse Dienst 1988: 4).

Nach einem Hinweis auf die Aufhebung des zeitlichen Kontinuums im Roman („Die Zeit zieht sich im Prisma des Textes zusammen, ereignet sich im Augenblick als Vergangenheit - Gegenwart - Zukunft"), bringt Bärbel Schulte abschließend die kreative Rolle des Lesers im Chasarischen Wörterbuch zur Sprache. Pavićs Roman sei, auch wenn sich „[m]it all diesen literarischen Verfahren [...] Autor und Text vom Leser zu isolieren" (Hanser Presse Dienst 1988: 5) scheinen, kein selbstgenügsames Buch. Im Gegenteil fordere es den als passiv charakterisierten zeitgenössischen Leser provokativ zur schöpferischen Mitarbeit auf.

Schultes Darstellung folgt in der Informationsbroschüre des Hanser Pressedienstes eine leicht gekürzte Fassung des bereits erörterten Artikels „Ökumenischer Traum“ von Zdzişlaw P. Gwozdz. Diesem schließen sich zwei Stellungnahmen mit ausgeprägtem Werbecharakter an, die aus zwei verschiedenen Perspektiven das Exzeptionelle des Chasarischen Wörterbuchs bezeugen sollen. Ein von Pavić zusammengestellter Kurzbericht bietet zunächst Einblick in den spektakulären Verlauf der Romanrezeption in Jugoslawien. Als literaturkritische Stimme, die den "Lexikonroman" werbend vorstellen soll, wird sodann ein Ausschnitt aus der ersten jugoslawischen Rezension des Chasarischen Wörterbuchs herangezogen, verfasst vom serbischen Dichter Raša Livada und erschienen in der Zeitschrift Delo zu Beginn des Jahres 1985. Die enthusmiasmierte Erstkritik von Livada huldigt dem Chasarischen Wörterbuch als Meilenstein der serbischen Literatur und, darüber hinaus, als Ausnahmeerscheinung der neueren literarischen Entwicklung überhaupt. Der Dichter ist fest überzeugt, der „Lexikonroman“ werde sogar eine fundamentale Verschiebung bzw. Wandlung gängiger rezeptioneller und interpretativer Horizonte provozieren:

Mit der Veröffentlichug dieses Buches ist die uns bekannte Literaturkritik endgültig an ihre Grenzen gelangt, da keine einzige literaturkritische Schule oder Methodologie in der Lage sein wird, die ganze Tragweite des Unterfangens von Pavić zu erklären. Das ist gleichzeitig auch der Augenblick, auf den wir seit langem warten, der Augenblick des Entstehens neuer Formen der Rezeption und der Interpretation des literarischen Werks. Vielleicht wird sich mit diesem Buch dennoch das ereignen, was sich nur mit den bedeutendsten ereignet - es wird niemals eine erschöpfende Interpretation finden, denn stets wird es eine Schicht mehr enthalten, eine Schicht tiefer greifen als irgendeine Interpretation" (Hanser Presse Dienst 1988: 5). 
Rückblickend betrachtet, scheint es, als hätten die wenigen frühen Berichte über das Chasarische Wörterbuch und die etwas ausführlichere Portraitierung von Schriftsteller und Werk in der Hanser Werbebroschüre ganz wesentlich die Entwicklung der Romanrezeption in der deutschsprachigen Tagespresse bestimmt. Diese offenbar suggestive Einwirkung der „ersten Worte“ auf Auswahl der Beschreibungsmittel, sowie auf Interpretations- und Wertungsmaßstäbe der Literaturkritik soll nun kurz umrissen werden.

Pavićs persönliche kulturideologische bzw. politische Positionen, wie sie der Autor bereits im ersten Presse-Artikel von Zdzişlaw P. Gwozdz artikuliert, gelten in der nachfolgenden Tageskritik als nahezu unumgängliche Interpretationsstütze und werden gerne direkt auf die Werkpoetik projiziert. Schon in den frühesten Stellungnahmen zum Chasarischen Wörterbuch in deutschsprachigen Medien greift Pavić weit über den unmittelbaren Horizont des Romans hinaus und beschreibt nicht nur den kulturhistorischen Rahmen, in den sein Buch zu situieren wäre, sondern bringt auch den aktuellen Zustand der von latenten Ost-West-Spannungen geprägten europäischen Kulturlandschaft zur Sprache. Im Mittelpunkt des frühen Presse-Gesprächs steht der Appell an die Bewahrung bzw. Rückbesinnung auf die europäische kulturelle Vielfalt. Im Zusammenhang damit verweist Pavić im selben Autemzug auf die enge Verflechtung zwischen Ideologie, Religion und Kultur. So kristallisiert sich bereits in dem frühen Zeitungsinterview auf Deutsch Pavićs kulturideologische Position klar heraus: Die Stärke der europäischen Kultur liegt in ihrer inneren Diversität bei gleichzeitiger Zusammengehörigkeit ihrer Teilkulturen. Die europäische Kultur ist eine vielfältige Einheit, mit gemeinsamem Quellgrund in der griechisch-römischen und judeo-christlichen Tradition. Zu jenem Zeitpunkt erblickt der Autor in der Ideologie die ultimative Bedrohung für eine so verstandene - vielfältige und zusammengehörige - gesamteuropäische Kultur. Die damalige ideologische Gespaltenheit Europas nach dem Muster Westen/Demokratie/Kapitalismus gegenüber Osten/Sozialismus/Kommunismus macht der Schriftsteller für eine entsprechende kulturelle Zweispaltung sowie für die potenzielle Gefahr der Tilgung kleiner europäischer Kulturen aus dem Kulturbewusstsein Europas verantwortlich. Im frühen Presse-Interview erklärt er die intensive kulturelle Vermittlung auf gesamteuropäischem Terrain zu jenem „ökumenischen“ Engagement, das die Vielfalt und Zusammengehörigkeit europäischer Kulturen bewahren soll, im impliziten Kampf gegen die Übermacht ideologischer und religiöser Agitationen und Teilungen. Der kulturellen Aktivität mutet Pavić also damals schon die Rolle der Spannungs- und Animositätsdämpfung zwischen Ost und West zu und verortet sein Chasarisches Wörterbuch innerhalb dieses kulturideologischen Paradigmas.

Die Problematik des europäischen Kulturbewusstseins behält Pavić auch später im Fokus. Leider wird das Thema nach wenigen Jahren 
aus tragischem Anlass aktualisiert, nämlich im Zuge der aufgekommenen national-religiösen Feindseligkeiten und der darauffolgenden kriegerischen Konflikte im ehemaligen Jugoslawien. Als er im Jahre 1991 in der deutschen Zeit nach den tieferen Kriegsursachen befragt wird, rückt Pavić wieder den potenziell fatalen Nexus zwischen Ideologie, Religion und Kultur in den Vordergrund. Doch in der veränderten politischen Konstellation erhalten seine Ausführungen andere Akzente. Hatte er die Bedrohung der kulturellen Vielfalt und Einheit Europas 1988 entlang der ideologischen Achse Kapitalismus-Kommunismus angesiedelt, sichtet er nun unter der Oberfläche der ideologischen Spaltung die Wirkung religiöser Animositäten als den eigentlichen Konfliktkeim. In Wahrheit werde in Jugoslawien ein Religionskrieg geführt. Als Abklang allgemeiner Verhältnisse manifestiere sich in diesem Krieg eine unterschwelige Ignoranz der westchristlichen Kultursphäre Europas gegenüber dem Ostchristentum. Die ideologische Skepsis des Westens gegenüber dem Kommunismus verdeckt Pavić zufolge den tiefer sitzenden Spaltungsgrund, der in der Abkehr des westlichen Bewusstseins von der orthodox-ostchristlichen Welt wurzelt. Gerade den Folgen dieser kulturellen und religiösen Ignoranz hätten die Serben die offene Feindseligkeit des Westens im Jugoslawienkrieg zu verdanken, eine Feindseligkeit der sie als kleines orthodoxes Volk ohne religiöse Protektion auf internationaler Ebene (im Gegensatz zum Katholizismus verfügt die Orthodoxie über keine gemeinsame kirchliche Organistation) schutzlos ausgeliefert gewesen seien (Vgl. Pavić, „Serbien, Byzanz und Europa“, 1991).

Führt man sich die erweiterte Semantik von Ökumene aus dem frühen Presse-Gespräch vor Augen, so kann man schließen, dass sich Pavić im Jahr 1988 nicht primär für religiöse, sondern eher für eine Art umfassendere „Kulturtoleranz“ zwischen dem Westen und Osten Europas, bei implizitem Kampf gegen ideologisches Blockdenken, stark macht. Die spätere Akzentverlagerung, wo nicht mehr in der Ideologie, sondern in der Religion das Trennende erkannt wird, bedeutet aber keine so große Perspektivenverschiebung wie es auf den ersten Blick scheinen mag. Hier wie dort platziert Pavić den Gedanken von der Opposition zweier Kultursphären Europas, die sich ihres gemeinsamen Ursprungs und ihrer prinzipiellen Zusammengehörigkeit besinnen sollten. Der westliche Kulturraum sei im Grunde vom Westchristentum dominiert, der östliche vom byzantinischen Erbe der Orthodoxie. Dass Pavić die religiöse Dominante dieses Kulturrisses im Jahre 1988 nicht erwähnt und sie zu jenem Zeitpunkt lieber auf die Ideologie verschiebt, lässt sich mit einer kontextbedingt entspannteren Urteilsposition des Schriftstellers erklären. Im friedlichen Kontext des Jahres 1988 ist Pavić sicherlich mehr an der Betonung des potenziell Gemeinsamen zwischen Ost und West gelegen. Eine stärkere Betonung tief reichender Ursachen der Divergenzen, die eine Folge von Jahrhunderte langen Entwicklungen in unterschiedlichen, religiös dominierten Kultursphären sind und so etwas wie das je- 
weilige kollektiv Unbewusste darstellen könnten, wäre im „Friedensjahr“ 1988 wohl nicht sehr förderlich und im Sinne von Pavićs Kulturökumene sogar kontraproduktiv gewesen.

Was Pavić dabei nicht explizit ausspricht, aber zwischen den Zeilen deutlich abgelesen werden kann, ist der Umstand, dass religiöse Standpunkte den Charakter ideologischer Positionen annehmen können. Man erinnere sich an dieser Stelle noch einmal daran, dass der früheste Hinweis auf das Chasarische Wörterbuch in einem deutschsprachigen Medium gerade im Konetxt der Polemik um die Grenzziehung zwischen religiösen und kulturhistorischen Phänomenen geschieht. Und mehr noch - der betreffende Artikel aus dem Jahr 1985 erhellt gerade jene Erscheinung, die Pavić im Zusammenhang mit dem Chasarischen Wörterbuch mit Nachdruck betont: die Gespaltenheit der Ost- und Westkirche, die hier bei der Kontroverse um die historische Rolle Kyrills und Methods in aller Deutlichkeit sichtbar wird. Wie gezeigt, interpretieren dabei die West- und die Ostkirche die historische Rolle der Mission Kyrills und Methods zu je eigenem Gunsten. Die westliche Perspektive schiebt mit der Betonung der religiösen Aspekte der Bekehrung der slawischen Völker die eigene Rolle bei der Missionierung der Slawen im Namen einer einheitlichen katholischen Kirche in den Vordergrund. Die Ostkirche besteht hingegen auf der umfassenderen kulturellen Bedeutung der Mission und der folglichen Eingliederung vieler slawischer Völker in den orthodox-byzantinischen Kulturraum. Vor dem Hintergrund dieser noch im Jahr 1985 erhitzt geführten Polemik zwischen West- und Ostkirche gewinnen Pavićs alarmierende Gedanken zu den religiösen Ursprüngen der europäischen Kulturspaltung an Überzugungskraft. Dass es in der zwischenkirlichen Auseinandersetzung über Jahrhunderte alte Ereignisse um keine theologischen Fragen geht, sondern um die Festigung des Machtstatus der West- bzw. Ostkirche, bringt die Polemik in nächste Nähe zum ideologischen Diskurs und veranschaulicht die oft schwer einzufangende Trennlinie zwischen Ideologie, Religion und kulturellem Engagement. Auf die Existenz dieser diffusen Trennlinie verweist auch Pavić mit seinen vielen Stellugnnahmen zur Problematik, unter anderem indem er die Akzente mal auf das Religiöse, mal auf das Ideologische setzt. Zusammenfassend bleibt festzuhalten: Die enge Verflechtung kultureller, religiöser, ideologischer und politischer Phänomene prägt die deutschsprachige Berichterstattung über das Chasarische Wörterbuch und seinen Autor seit den frühesten Erwähnungen in der Tagespresse und bleibt auch im Folgenden sehr charakteristisch für den zeitungskritischen Diskurs.

Pavić bringt also bereits im frühen Presse-Gespräch seine kulturhistorischen und -ideologischen Gedanken in engsten Zusammenhang mit den eigenen Interpretations-vorschlägen zum Chasarischen Wörterbuch. Die deutschsprachige Tageskritik macht es ihm gewisserweise nach. Dasselbe Gefüge aus Fragmenten kulturideologischer Einstellung und hermeneutischen Ansätzen ist in der Folge immer wieder auch in 
den einzelnen Romanrezensionen anzutreffen. Vor allem die Dekodierung der chasarischen Metapher verläuft später oft entlang dieses frühen Deutungschemas. Der Auslegung der chasarischen Identitätsproblematik als einer Suche nach dem kulturellen Selbstverständnis der Serben innerhalb der byzantinischen Tradition, wie von Pavić im Presse-Gespräch skizziert, wird später zwar nahezu keine Beachtung geschenkt. Hingegen besteht die Kritik sehr gerne auf dem gesellschaftspolitisch profiliertem Subtext des Romans, oft in Anlehnung an die frühen Erläuterungen des Schriftstellers selbst. So wird Pavićs Phrase „kleines Volk zwischen großen Ideologien, Religionen und Mächten, das diese Ideologien und Religionen sowie die Macht nicht teilt" in der Tageskritik vielerorts wörtlich oder nahezu wörtlich übernommen. Sie gilt als willkommene Autointerpretation des Schriftstellers, wenn es darum geht, das Verschwinden der Chasaren von der Weltbühne als Sinnbild der existentiellen Bedrohung kleiner Völker (respektive der Serben) im Kontext sie übertreffender historischer Gegebenheiten auszulegen.

Auch die Repräsentation des ökumenischen Gedankens religiöser Toleranz wird dem Chasarischen Wörterbuch seitens der späteren Tageskritik immer wieder gutgeschrieben, erweitert um das Aufzeigen von Analogien zu Lessings "Ringparabel“ aus Nathan dem Weisen. Diese ethische Lesart bzw. die Toleranzthematik bleiben auch weiterhin im Blickfeld der Zeitungskritik, erfahren aber innerhab der akademischen Diskussion eine weitaus komplexere Behandlung.

Ähnlich steht es um die im engeren Sinne poetischen Dominanten des Chasarischen Wörterbuchs. Von Bärbel Schulte und Zdzişlaw P. Gwozdz gezogene Parallelen zwischen Pavić und Umberto Eco bleiben druchgehend im Blickpunkt der zeitungskritischen Beiträge, wobei später auch Autoren wie Jorge Luis Borges und Julio Cortázar apostrophiert werden.

Besonders auffallend ist der sehr frühe Hinweis auf die genremäßige Unbestimmtheit des Chasarischen Wörterbuchs, oder, besser gesagt, die Nachwirkung dieses Hinweises in der Kritik. Noch 1986 bezeichnet Vladimir Ulrich das Werk als „Abenteuerroman, eine Sammlumg von Erzählungen, Märchen und Legenden, ein Gedichtband, eine historische Studie, ein kabbalistisches Handbuch - und vieles mehr" (Ulrich 1986). Ähnliches findet sich auf dem Umschlag der ersten deutschen Ausgabe des Chasarischen Wörterbuchs. Dort wirbt eine stichwortartig gegliederte Kurzbeschreibung für die Gattungsheterogenität des Werks. Beim Stichwort „Das Buch“ heißt es: „Abenteuerroman, Liebesroman, historischer Roman, Sammlung von Versen, Puzzle, Traumbuch, Kriminalroman, Rätselspiel, Denksportaufgabe, Zauberwürfel und Vexierbild“ (Pavić 1988). Die Taschenbuchausgabe des DTV-Verlags bringt auf der ersten Innenseite eine etwas kürzere und leicht geänderte Darstellung: „Abenteuerroman, Liebesroman, historischer Roman, Krimi, kabbalistisches Handbuch, Traumbuch, Denksportaufgabe" (Pavić, Das Chasarische Wörterbuch, 1991). Es ist nicht klar, ob die Klappentexte der deutschen Ausgaben auf 
die Beschreibung von Vladimir Ulrich aus dem Jahr 1986 zurückgehen, doch alles deutet darauf hin. Interessanter ist jedoch der Nachhall dieser Qualifikationen in der späteren Tageskritik. Auffallend oft stößt man in den einzelnen Beiträgen auf eine Anzahl (fast) identischer Gattungszuordnungen, die auf eine direkte Übernahme jener von den Klappentexten bzw. aus Ulrichs Frührezension stammenden Charakterisierung des Romans schließen lassen. Immer wieder tauchen die gleichen Genredefinitionen, häufig auch in einer sehr ähnlichen Konstellation, auf: „Kriminalroman“, "Abenteuerroman“, „Liebesroman“, „kabbalistisches Handbuch“, „halbkabbalistisches Handbuch“, „historischer Roman“, „Gedichtband“, „Puzzlespiel“, „Traumbuch“, „Vexierbild“ u. a. ${ }^{5}$ Als einzige unter den Rezensenten verweist dabei Karin Rahn auf den Buchumschlag der Erstausgabe als Quelle dieser teilweise sehr exotisch anmutenden Gattungsbestimmungen (Vgl. Rahn 1988), während alle anderen die oben erwähnten Definitionen offenbar lediglich schablonenhaft übernehmen. Somit kommt die Frage auf, ob die gattungsmäßige Diversität bei einer ganzen Reihe von Kritikern tatsächlich wahrgenommen oder eher als vorgefertigte Darstellung Vladimir Ulrichs bzw. der beiden Verlage übernommen wird. Dies ist umso wahrscheinlicher, als von den genannten Qualifikationen viele eher der freien, leicht poetisierenden Darstellungsweise eines Werbetextes entsprechen, und einer strengeren poetologischen Analyse wohl nicht standhalten würden. So sind die Charakterisierungen des Romans als "Gedichtband“ oder „(halb)kabbalistisches Handbuch“ weniger als ernst zu nehmende Gattungseigenschaften und mehr als rhetorische Ausschmückungen aufzufassen, die die breite ästhetische Skala des Romans unterstreichen sollen. Dichtung im engeren Sinne kommt im Werk nur an einigen wenigen Stellen vor. Doch weder diese noch einzelne weitere poetisierende Passagen oder etwa die metaphernreiche Sprache machen das Chasarische Wörterbuch zu einem Gedichtband in einem genremäßig verantwortlichen Sinn.

Wie in Bärbel Schultes obiger Darstellung wird Phantastik, trotz gleichzeitigem Bestehen auf der Gattungsheterogenität des Werks, auch weiterhin als das dominante Wesensmerkmal des Chasarischen Wörterbuchs, ja sogar als differentia specifica von Pavićs literarischem Schaffen überhaupt, erkannt und anerkannt werden. Der Fokus liegt dabei auf der phantastischen Unterwanderung von empirischer Wirklichkeit und den

5 Carlo Bernasconi reiht die folgenden Genrezuordnungen aneinander: „Abenteuerroman, Liebesroman, kabbalistisches Handbuch, Traumbuch, Kriminalroman" (Bernasconi 1988); In der anonymen Rezension der Zeitschrift Semit, deren Text in Anspielung auf die Struktur des Chasarischen Wörterbuchs die Form der Lexikoneinträge nachahmt, heißt es zum Stichwort „das Buch“: „ein Abenteuerroman, ein Liebesroman, ein historischer Roman, eine Sammlung von Erzählungen und Versen, ein kabbalistischer (sic!) Handbuch, ein Puzzle, ein Traumbuch, ein Kriminalroman, ein Rätselspiel, eine Denksportaufgabe, ein Zauberwürfel, ein Vexierbild“ (Anonym 2 1988); Laut Günther Fässler ist das „wunderschön ausgestattete Lexikon [...] Krimi und religiöser Roman, Vexierbild, Puzzle und Traum“ (Fässler 1988); Und auch bei Gerd-Klaus Kaltenbrunner stößt man auf das gleiche Muster - das Werk sei „zugleich Chronik, Abenteuer-, Liebes- und historischer Roman, aber auch Traumbuch, Puzzlespiel, Kreuzworträtsel, Domino, Vexierbild“ (Kaltenbrunner 1988). 
somit „freigesetzten“ Realitätsebenen des Unbewussten, Onirischen und Irrationalen. Metaphysisches und Okkultes als Erscheinungsformen des Phantastischen (oder eventuell als eigenständige Romanebenen) werden hingegen schon bei Gwozdz und Schulte ausgespart und auch in der nachfolgenden tageskritischen Rezeption weniger beachtet.

Relativ großen Anklang in der Zeitungskritik findet später auch Pavićs rezeptionsbezogen relevantes Konzept des "schöpferischen Lesers“, das ebenfalls bereits im Informationsheft des Hanser Verlags aufgeworfen worden war.

Die Gedanken zum Ausnahmecharakter des Chasarischen Wörterbuchs, die schon sein erster Rezensent Raša Livada vorformuliert und das Hanser Informationsheft übernommen hat, fallen in der Tageskritik ebenfalls auf fruchtbaren Boden. Immer wieder äußert diese die Überzeugung, der „Lexikonroman“ sprenge alle tradierten Gattungsformen und poetischen Konzeptionen, und entziehe sich damit sämtlichen überlieferten Interpretationsmethoden und -zugängen.

Im abschließenden Summieren der Eindrücke kommt die Frage auf, wie man die Tatsache interpretieren soll, dass sämtliche Urteile der Tageskritik über das Chasarische Wörterbuch, bei allem Facettenreichtum der späteren Rezeption, bereits in den wenigen der Publikation des Romans vorangehenden Artikeln und einer Werbebroschüre des Hanser Verlags vorformuliert gewesen sind. Alles deutet darauf hin, dass die große Mehrheit der Rezensenten die in den Frühberichten zu Werk und Autor vorgegebenen Koordinaten in ihr eigenes Beschreibungs- und Bewertungsinventar aufgenommen hat. Sicherlich reicht ein Fallbeispiel bei weitem nicht aus, um aus dessen Eigentümlichkeiten rezeptionsästhetisch relevante Konsequenzen zu ziehen. Doch erlaubt es dieses Beispiel zumindest rezeptionstheoretische Fragestellungen zu formulieren. Aus einer wohlgesinnten Perspektive heraus könnte man die Vermutung aufstellen, dass es in der Tageskritik aufgrund gleicher und ähnlicher Eindrücke zur breiten Übereinstimmung der Gemüter über das Chasarische Wörterbuch gekommen ist, zur spontanen harmonischen Einstimmigkeit der interpretativen Gemeinschaft, deren Mitglieder von sich aus, unabhängig von äußeren Einwirkungen, zu gleichen Einsichten über das Werk gekommen sind. Doch ein skeptischerer Blick erspäht unter dem Bild allgemeiner Einmütigkeit eine Art literaturkritischen Konformismus, der sich bei aller späteren Differenzierung und rhetorischen Anreicherung der Urteile, im Grunde an die vorformulierten Bewertungen der Erstrezensionen verlässt. Eine letzte Vermutung wäre schließlich noch imstande, die im vorherigen Satz angezweifelte Ehre des Literaturkritikers zu retten. In der immensen Anzahl teilweise hymnisch geschriebener Rezensionen findet sich eine breite Palette von sehr plausiblen Einschätzungen, treffend gewählten Darstellungen und, vor allem, Impressionen, die auf ein starkes unmittelbares Leseerlebnis der Rezensenten schließen lassen. Vor allem die Ausführungen über den Ausnahmecharakter des „Lexi- 
konromans", die regelrechten Beichten über die eigene diskursive Unbeholfenheit vor dem Wunder Chasarisches Wörterbuch hören sich in den Ohren des Lesers ehrlich an. Dass man sich bei der Wiedergabe zweifellos tiefer Eindrücke lieber auf Festgelegtes verlässt anstatt eigene Schritte zu wagen, könnte im Fall von Pavićs Roman tatsächlich mit der Scheu vor der Versprachlichung des unsagbar Scheinenden zusammenhängen. In dieser leicht mystisch anmutenden Atmosphäre entwickeln offenbar die ersten „tapferen“ Worte, die es gewagt haben, das Unfassbare fassbar zu machen, eine Suggestivkraft, der man sich schwer zu entziehen vermag.

\section{LITERATURVERZEICHNIS}

Anonym 1. „Bärbel Schulte, Pavić-Übersetzerin.“ Mittelbayerische Zeitung (Regensburg) 4.5. 1988.

Anonym 2. [Das Chasarische Wörterbuch]. Semit Nr. 0, Jg 1. 1988.

Bernasconi, Carlo. „Ein Streifzug durch das verrückteste Buch des Jahres 1988." Schweizer Manager April 1988.

Цидилко, Весна. „О књижевности у сенци политике или Милорад Павић на немачком.“" Лейойис Майище срӣске 182. 4 (2006): 609626.

Fässler, Günther. „Ein Traum von einem Buch zwischen den Wörtern.“ Der Landbote 13. 8. 1988.

Gwozdz, Zdzişlaw P. „Ökumenischer Traum.“ Die Presse 30./31. 1. 1988.

(Hanser Presse Dienst): Informationen zu Milorad Pavić und „Das Chasarische Wörterbuch, Lexikonroman in 100.000 Stichworten“, München: Carl Hanser Verlag, 1988.

Kaltenbrunner, Gerd-Klaus. „Literarisches Verwirrspiel aus Jugoslawien.“ Welt am Sonntag 25. 9. 1988.

Kroll, Walter. „Das Kulturmodell der Südslaven im europäischen Kontext.“ Uni-Report der Universität Mannheim 1.

Maier, Viktor. „Der heilige und der historische Method.“ Frankfurter Allgemeine Zeitung 21. 9. 1985.

Pavić, Milorad. Das Chasarische Wörterbuch. München, Wien: Carl Hanser Verlag, 1988.

Pavić, Milorad. Das Chasarische Wörterbuch. München: Deutscher Taschenbuch Verlag, 1991.

Pavić, Milorad. „Serbien, Byzanz und Europa“. Die Zeit 26. 7. 1991.

Павић, Милорад. Роман као gржава и gруїи оїлеgи. Београд: Плато, 2005.

Rahn, Karin. „Nanu - ein Lexikonroman?“ Landshuter Zeitung 8. 4. 1988.

Ulrich, V(ladimir). „Verwirrender Literatur-Leckerbissen.“ Mittelbayerische Zeitung 12. 12. 1986. 
Ulrich, V(ladimir). „Skurrile Reise durch einen literarischen Kosmos.“ Deutsche Tagespost 21. 1. 1989.

Wittmann, Mirjana. „Schreiben wie ein Schmuggler.“ Deutsches Allgemeines Sonntagsblatt 19. 6. 1988.

\section{Милица Мустур}

Рецейцијски тіse-еn-abyте. Рана крийика Хазарског речника на немачком

\section{Резиме}

Са имплицитним погледом на питања од теоријског значаја, у раду се анализира најранија фаза у пријему Хазарскоі̄ речника Милорада Павића на немачком говорном подручју. У фокусу истраживања стоје извештаји о роману и његовом аутору у немачким штампаним медијима објављени пре публикације немачког превода дела, уз поглед на каснији развој рецепције у оквиру новинске, дневне критике. Детаљан приказ садржаја ове ране рецепцијске фазе показује да су већ тада, пре изласка из штампе Хазарскоі речника на немачком језику, формулисани безмало сви критички судови о роману као и слика личног и ауторског профила писца карактеристични за целокупан каснији пријем романа у дневној критици. Рад се окончава питањем о вредности ових закључака за сагледавање општих механизама књижевне рецепције, тј. за могућа уопштавања у домену естетике рецепције.

Кључне речи: Милорад Павић, Хазарски речник, немачко говорно подручје, механизми књижевне рецепције, историја рецепције, естетика рецепције 